\title{
THE WAVE EQUATION IN EXTERIOR DOMAINS
}

\author{
BY PETER D. LAX $^{1}$ AND RALPH S. PHILLIPS ${ }^{2}$ \\ Communicated by Lipman Bers, October 26, 1961
}

This note deals with solutions of the wave equation in three dimensions in the exterior of a finite number of smooth obstacles, on whose boundaries the solution is subject to boundary conditions of the form $u=0$ or $u_{n}=\sigma u, \sigma$ a non-negative function. We shall show that every such solution of finite energy propagates eventually out to infinity and behaves asymptotically like a free space solution.

TheOREM I. For every nonzero solution there exists a positive constant $d$ less than the total energy of the solution such that given any bounded domain, there is a time at which the energy contained in the exterior of this domain exceeds $d$.

SkETCH OF PROOF. Suppose the theorem is false for some $u$; then given any positive $\epsilon$ there exists a bounded domain such that the energy contained in its exterior is less than $\epsilon$ for all time. Assume that $u$ is a smooth function; ${ }^{3}$ applying the law of conservation of energy to $u_{t}$ and using standard estimates for $u_{x x}$ in terms of $\Delta u$, we see that the square integral of the second partial derivatives of $u$ over the exterior of the obstacles is uniformly bounded for all time. Let $\left\{t_{n}\right\}$ be an arbitrary sequence of numbers; using the Rellich selection theo$\mathrm{rem}^{4}$ and a diagonal process we can choose a subsequence such that the first partial derivatives of $u$ form a Cauchy sequence in the square integral sense over any bounded domain in $x$-space. Since we have supposed that the energy contained outside bounded domains is uniformly small for all $t$, it follows that $\left\{u\left(t_{n}\right)\right\}$ is a Cauchy sequence in the energy norm over the whole exterior. This shows that $u(t)$ is a vector-valued almost periodic function of $t$. By the main theorem of a.p. functions $u(t)$ is a superposition of exponentials:

$$
u \approx \sum a_{j}(x) e^{i \omega_{j j} t}
$$

From the mean value expression for the coefficient $a_{j}$ it follows that it is a solution of the reduced wave equation $\Delta a+\omega_{j}^{2} a=0$. But according to a theorem of Rellich, ${ }^{5}$ the reduced wave equation has for $\omega_{j} \neq 0$

${ }^{1}$ Sloan Fellow.

${ }^{2}$ Sponsored by the National Science Foundation, contract NSF-G16434.

${ }^{3} u$ can be made smooth by mollifying it with respect to $t$.

${ }^{4}$ F. Rellich, Göttinger Nachrichten, 1930.

5 F. Rellich, Jber. Deutsch. Math. Verein. 53 (1943), 57-65. 
no nonzero solution which vanishes near infinity; so all Fourier coefficients with $w_{j} \neq 0$ vanish. This shows that $u$ is a time independent harmonic function; since $u$ satisfies the imposed boundary conditions it follows that $u=0$.

Another application of the selection principle yields this

Corollary. Given any bounded domain $D$ and any $\epsilon$, there exist arbitrarily large values of $t$ for which the energy contained in $D$ at time $t$ is less than $\epsilon$.

We define a detached solution of the mixed problem as one which vanishes in a cone $|x| \leqq t-t_{0}$ for some $t_{0}$. According to Huygens' principle, any solution of the wave equation in free space whose Cauchy data at $t=0$ have compact support becomes detached for $t$ large enough.

Theorem II. The Cauchy data of detached solutions are dense in the space of all Cauchy data relative to the energy norm.

Sketch of PROof. Let $\phi$ denote Cauchy data which are orthogonal in the energy norm to the Cauchy data of all detached solutions. Let $v(t)$ be the solution of the mixed problem with Cauchy data equal to $\phi$ at $t=0$. It is easy to show that the Cauchy data of $v$ at any other time have the same orthogonality property. Denote by $w_{s}$ a solution of the wave equation in free space whose Cauchy data at $t=s$ agree with those of $v$ outside the obstacles. Using the orthogonality of $v$ to the detached solutions constructed above one shows easily that $w_{s}$ is zero in the backward cone $|x|<s-t-r$, where $r$ is the radius of a sphere around the origin which contains the obstacles. Thus for $t<s-2 r, w_{s}$ is a solution of the mixed problem as well.

Since $v$ and $w_{s}$ have the same Cauchy data at $t=s$ outside the obstacles, they are equal in the domain of dependence $|x|>|t-s|+r$. Applying the law of conservation of energy to $v-w_{s}$ we see that the energy of $v$ at $t=0$ inside the sphere of radius $s-r$ is bounded by the energy of $v-w_{s}$ at time $t=s-2 r$ inside the sphere of radius $3 r$. This is bounded by a constant times the energy of $v$ at time $t=s$ inside the sphere of radius $5 r$. But according to the corollary of Theorem I there exist arbitrarily large values of $s$ for which the latter quantity is arbitrarily small. This shows that $v$ has zero energy, q.e.d.

Theorem II shows that any solution with finite energy can be approximated by detached solutions. Thus for any solution the amount of energy contained in a given bounded set tends to zero with increasing $t$. Previously, Cathleen S. Morawetz ${ }^{6}$ has shown for the ex-

\footnotetext{
6 Comm. Pure Appl. Math. (1961).
} 
terior of a single star-shaped obstacle and for any solution which satisfies the boundary condition $u=0$ with Cauchy data at $t=0$ vanishing outside a sphere $S$, that the energy contained in sphere $D$ is less than ${ }^{7}$ const. $E / t$, where $E$ is the total energy and the value of the constant depends only on the radii of $S$ and $D$. We wish to point out that such a quantitative result about energy decay can hold only if the obstacle satisfies the following geometric condition: No two boundary points of it form a segment exterior to the obstacle and perpendicular to it at both endpoints. For a narrow high frequency beam directed along such a segment would be reflected back and forth for a length of time proportional to the reciprocal of wave length.

If one regards the presence of the obstacle as a perturbation of free space and applies the usual scattering theory formalism, ${ }^{8}$ then it follows from Huygens' principle in free space that the so-called wave operators exist, and it follows further from Theorem II that they are unitary. This proves

THEOREM III. The perturbed and unperturbed problems are unitarily equivalent; in particular, the spectrum of the wave equation generator is unaffected by the presence of obstacles.

Added in proof. Recently we have succeeded in deriving Theorem II without making use of Huygens' principle, by using solutions which are superpositions of plane waves of finite width. Thus the results of this note can be extended to a large class of hyperbolic equations.

Institute of Mathematical Sciences, New York University aNd STANFORD UNIVERSITY

7 In a more recent paper, Morawetz has shown that energy decays like $1 / t^{2}$.

${ }^{8}$ See S. T. Kuroda, Perturbation of continuous spectra by unbounded operators. I, J. Math. Soc. Japan 11 (1959), 247-262; or J. M. Jauch, Theory of the scattering operators, Helv. Phys. Acta 31 (1958), 127-158.

\section{ADDENDUM TO ON THE EIGENVALUE OF POSITIVE OPERATORS ${ }^{1}$}

BY GIAN-CARLO ROTA

Two important assumptions on the operator $P$ in the Theorem stated in this note were omitted by a clerical error. They are

(a) the operator $P$ is positive ( $P f \geqq 0$ if $f \geqq 0$ ).

(b) the number $\alpha$ is of absolute value one.

These assumptions are stated in the informal discussion at the beginning of the note, and are used throughout in the proof.

${ }^{1}$ See volume 67, no. 6 (November 1961) pp. 556-558 of this Bulletin. 